\title{
Oxidative stress mediates nephropathy in type la glycogen storage disease
}

\author{
Wai Han Yiu', Paul A Mead', Hyun Sik Jun, Brian C Mansfield and Janice Y Chou
}

Glycogen storage disease type la (GSD-la) patients, deficient in glucose-6-phosphatase- $\alpha$, manifest disturbed glucose homeostasis with long-term renal disease. We have previously shown that renal fibrosis in GSD-la is mediated by the angiotensin/transforming growth factor- $\beta 1$ (TGF- $\beta 1$ ) pathway, which also elicits renal damage through oxidative stress. In this study, we further elucidate the mechanism of renal disease by showing that renal expression of Nox- 2 , p22 ${ }^{\text {phox }}$, and p $47^{\text {phox }}$, components of NADPH oxidase, are upregulated in GSD-la mice compared with controls. Akt/protein kinase B, a downstream mediator of angiotensin II and TGF- $\beta 1$, is also activated, leading to phosphorylation and inactivation of the Forkhead box $\mathrm{O}$ family of transcription factors. This in turn triggers downregulation of superoxide dismutase and catalase (CAT) activities that have essential roles in oxidative detoxification in mammals. Renal oxidative stress in GSD-la mice is shown by increased oxidation of dihydroethidium and by oxidative damage of DNA. Importantly, renal dysfunction, reflected by elevated serum levels of blood urea nitrogen, reduced renal CAT activity, and increased renal fibrosis, is improved in GSD-la mice treated with the antioxidant drug tempol. These data provide the first evidence that oxidative stress is one mechanism that underlies GSD-la nephropathy.

Laboratory Investigation (2010) 90, 620-629; doi:10.1038/labinvest.2010.38; published online 1 March 2010

KEYWORDS: Akt/protein kinase B; Forkhead box O; glycogen storage disease type la; NADPH oxidase; renal oxidative stress; catalase

Glycogen storage disease type Ia (GSD-Ia, MIM232200) is an autosomal recessive disorder caused by a deficiency in the endoplasmic reticulum-bound glucose-6-phosphatase- $\alpha$ (G6Pase- $\alpha$, also known as G6PC) that catalyzes the hydrolysis of glucose-6-phosphate (G6P) to glucose and phosphate in the terminal step of gluconeogenesis and glycogenolysis. ${ }^{1}$ GSD-Ia patients manifest a phenotype of disturbed glucose homeostasis characterized by fasting hypoglycemia, hepatomegaly, nephromegaly, hypercholesterolemia, hypertriglyceridemia, hyperuricemia, lactic acidemia, and growth retardation. ${ }^{1}$ Long-term complications include osteoporosis, gout, pulmonary hypertension, renal disease, and hepatocellular adenomas. Currently, there is no cure for GSD-Ia, but many of the disease symptoms can be managed or improved using dietary therapies $^{2,3}$ that maintain normoglycemia. This strategy enables patients to attain near-normal growth and pubertal development, with fewer complications as they age. Chronic renal disease was recognized as a major complication of GSD-Ia in the late 1980s. Renal biopsies of GSD-Ia patients revealed tubular atrophy, focal segmental glomerulosclerosis, and interstitial fibrosis. ${ }^{4-6}$ As dietary therapies delay the clinical onset of renal disease, the renal insufficiency characterized by glomerular hyperfiltration, hypercalciuria, hypocitraturia, and urinary albumin excretion still occur in metabolically compensated GSD-Ia patients. ${ }^{7,8}$

In previous studies of the biology and pathophysiology of GSD-Ia, we generated a G6Pase- $\alpha$-deficient mouse strain that faithfully mimics the human GSD-Ia disorder. ${ }^{9}$ Using the GSD-Ia mice, we have initiated studies on the role of the angiotensin (Ang) system ${ }^{10,11}$ in the development of renal disease. ${ }^{12}$ We have shown that the components of the Ang system and transforming growth factor- $\beta 1$ (TGF- $\beta 1),{ }^{11,13}$ that mediate most of the profibrogenic effects of Ang II, are upregulated in GSD-Ia mice relative to their unaffected littermates. ${ }^{12}$ We have also shown that the resulting renal fibrosis is characterized by a marked increase in the synthesis and deposition of extracellular matrix proteins in the renal cortex, and by the renal pathology of tubular basement

\footnotetext{
Section on Cellular Differentiation, Program on Developmental Endocrinology and Genetics, National Institute of Child Health and Human Development, National Institutes of Health, Bethesda, MD, USA

Correspondence: Dr JY Chou, PhD, Program on Developmental Endocrinology and Genetics, National Institutes of Health, Building 10, Room 9D42, NIH, 10 Center Drive, Bethesda, MD 20892-1830, USA.

E-mail: chouja@mail.nih.gov

${ }^{1}$ These authors contributed equally to this work.

Received 21 October 2009; revised 28 December 2009; accepted 29 December 2009
} 
membrane thickening, tubular atrophy, tubular dilation, and multifocal interstitial fibrosis. ${ }^{12}$ These results suggest that one mechanism underlying the nephropathy in GSD-Ia is the Ang/TGF- $\beta 1$ pathway-mediated renal fibrosis. However, renal damage goes beyond fibrosis, and in this study we have investigated additional mechanisms that may impact nephropathy in GSD-Ia.

Both Ang $\mathrm{II}^{14,15}$ and TGF- $\beta 1^{16}$ can mediate renal damage through oxidative stress ${ }^{17-19}$ by promoting increased reactive oxygen species (ROS) production in non-phagocytic cells by activating NADPH oxidases. The NADPH oxidases ${ }^{20,21}$ are multi-protein complexes that catalyze the reduction of molecular oxygen to superoxide anion, which in turn can produce damaging ROS. In addition, Ang $\mathrm{II}^{22,23}$ and TGF- $\beta 1^{24,25}$ repress ROS detoxification by activating signaling through Akt/protein kinase B. ${ }^{26}$ Activated Akt phosphorylates and inactivates the Forkhead box O (FoxO) family of transcription factors, ${ }^{27,28}$ thereby repressing the expression of the antioxidant enzymes, superoxide dismutases (SODs) ${ }^{29,30}$ and catalase (CAT). ${ }^{31}$ SODs convert superoxide anion to hydrogen peroxide, thereby preventing the formation of highly aggressive compounds such as peroxynitrite and hydroxyl radical. ${ }^{29,30}$ CAT reduces hydrogen peroxide to water and oxygen. ${ }^{31}$ The result is that activation of Ang II and TGF- $\beta 1$ renders the cells more vulnerable to oxidative stress.

The kidney expresses all of the components of the phagocyte NADPH oxidase (gp91 ${ }^{\text {phox }}$ or Nox-2) and the NADPH oxidase homologs Nox-1 and Nox- $4 .{ }^{21}$ The phagocyte enzyme consists of membrane-associated flavocytochrome catalytic subunits, Nox-2 and p $22^{\text {phox }}$ that interact with various cytosolic proteins, including the regulatory subunit $\mathrm{p} 47^{\text {phox }}$. Moreover, the Akt/FoxO signaling pathway has a role in renal oxidative stress by suppression of the antioxidant $\mathrm{SOD}^{25}$ or $\mathrm{CAT}^{32}$ We propose that oxidative stress is another potential mechanism for renal damage in GSD-Ia. In this study, we show that an enhanced renal oxidative stress occurs in GSD-Ia mice, reflected by the increase in the expression of NADPH oxidase and the activation of the Akt/FoxO pathway-mediated suppression of SOD and CAT.

\section{MATERIALS AND METHODS G6Pase- $\alpha$-Deficient GSD-la Mice}

All animal studies were conducted under an animal protocol approved by the NICHD Animal Care and Use Committee. A glucose therapy, that consists of intraperitoneal injection of $25-100 \mu \mathrm{l}$ of $15 \%$ glucose, every $12 \mathrm{~h}$, was initiated on the first post-natal day for GSD-Ia mice, as described previously. ${ }^{9}$ Mice that survived weaning were given unrestricted access to Mouse Chow (Zeigler Bros, Gardners, PA, USA).

\section{Quantitative Real-Time RT-PCR, Western Blot, Serum, and Phenotype Analyses}

Total RNAs were extracted from the kidneys using the TRIzol Reagent (Invitrogen Life Technologies, Carlsbad, CA, USA). The mRNA expression was quantified by real-time RT-PCR, in triplicate, in an Applied Biosystems (Foster City, CA, USA) 7300 Real-Time PCR System using the gene-specific TaqMan Gene Expression Assays and then normalized to $\beta$-actin RNA. The following TaqMan Gene Expression Assays were used: CAT, Mm00437992_m1; SOD1, Mm01344233_g1; SOD2, Mm00449726_m1; SOD3, Mm00448831_s1; and $\beta$-actin, Mm00607939_s1. Data were analyzed using the SDS v1.3 software (Applied Biosystems) and expression levels normalized against the level of the appropriate transcript in a wild-type mouse, which was arbitrarily defined as 1 .

The kidney tissues were lysed in a sample buffer containing $10 \mathrm{mM}$ Tris- $\mathrm{HCl}, \mathrm{pH} 6.8,2 \%$ SDS, and $10 \%$ glycerol and the lysates were resolved by electrophoresis through a $10 \%$ polyacrylamide-SDS gel and trans-blotted onto polyvinylpyrrolidene fluoride membranes (Millipore, Bedford, MA, USA). The membranes were incubated with a mouse monoclonal antibody against $\mathrm{gp} 91^{\text {phox }}$ (BD Pharmingen, San Diego, CA, USA), p-Akt-Ser473 (Cell Signaling, Danvers, MA, USA), or $\beta$-actin (Santa Cruz Biotechnology, Santa Cruz, CA, USA), or a rabbit polyclonal antibody against CAT or SOD1 (Abcam, Cambridge, MA, USA), against SOD2 or SOD3 (Stressgen, BC, Canada), against Akt, FoxO1 (H-128), FoxO3a (H-144), p-FoxO1-Ser256/p-FoxO4-Ser193, or p-FoxO1-Thr24/p-FoxO3a-Thr32, all from Cell Signaling, against $\mathrm{p}^{\text {phox }}(\mathrm{H}-195), \mathrm{p} 22^{\text {phox }}$ (FL-195), or Nox-4, all from Santa Cruz Biotechnology. Membranes were then incubated with the appropriate horseradish peroxidase-conjugated second antibody (Pierce, Rockford, IL, USA) and the immunocomplex was visualized using the Immobilon western chemiluminescent HRP substrate (Millipore). At least three separate experiments were conducted for each protein, in which each mouse was assessed individually.

Blood samples were collected from the tail vein and blood urea nitrogen (BUN) levels were analyzed using the Infinity Urea Reagent (Thermo Electron, Louisville, CO, USA). For hematoxylin and eosin (H\&E), Masson's trichrome, and van Gieson staining, tissues were preserved in $10 \%$ neutralbuffered formalin, embedded in paraffin, and sectioned at $5-\mu$ thickness. The stained sections were visualized using the Axioskop2 plus microscope and the AxioVision 4.5 software (Carl Zeiss, Thornwood, NY, USA). Quantification of renal fibrosis was performed on the longitudinal sections stained with van Gieson, and the red stained area was converted into pixel density units using the Adobe Photoshop CS3 (Adobe System Incorporated, San Jose, CA, USA). Pictures of the kidneys were stitched together using the Photomerge feature of Adobe Photoshop. After stitching, the pelvic areas were cropped out and renal fibrosis was measured only at the cortical and medullar regions.

\section{Immunohistochemical and Immunofluorescence Analyses}

Mouse kidneys were snap-frozen, embedded in O.C.T. (Sakura Finetek, Terrance, CA, USA), and sectioned at $5 \mu$ thickness. For immunohistochemical analysis, the tissue 
sections were prepared by quenching the endogenous tissue peroxidases with $0.3 \%$ hydrogen peroxide in $70 \%$ methanol for $30 \mathrm{~min}$. The endogenous avidin/biotin was blocked using the Avidin/Biotin Blocking Kit obtained from Vector Laboratories (Burlingame, CA, USA), and the sections were incubated with a rabbit polyclonal antibody against p-FoxO3-Ser253 (Cell Signaling) or a mouse monoclonal antibody against 8-hydroxy-2'-deoxyguanosine (8-OHdG, OXIS International, Foster City, CA, USA). The sections were then incubated with biotinylated anti-rabbit IgG or biotinylated anti-mouse IgG (Vector Laboratories) and the immune complexes were detected using an $\mathrm{ABC}$ kit and $\mathrm{DAB}$ Substrate from Vector Laboratories, according to the manufacturer's instructions. The sections were finally counterstained with hematoxylin before mounting.

For immunofluorescence detection of superoxide, the frozen kidney sections were equilibrated for $30 \mathrm{~min}$ at $37^{\circ} \mathrm{C}$ in Krebs' HEPES buffer (20 mM HEPES, pH 7.4, $128 \mathrm{mM}$ $\mathrm{NaCl}, 2.5 \mathrm{mM} \mathrm{KCl}, 2.7 \mathrm{mM} \mathrm{CaCl}_{2}, 1 \mathrm{mM} \mathrm{MgCl}_{2}$, and $16 \mathrm{mM}$ glucose). Then, the tissue sections were incubated in a light protected humidified chamber with Krebs' HEPES buffer containing $2 \mu \mathrm{M}$ dihydroethidium (DHE) for $30 \mathrm{~min}$ at $37^{\circ} \mathrm{C}$, and washed for 5 min with PBS. Dihydroethidium is a hydrophobic uncharged compound that is able to cross intracellular membrane and on oxidation becomes positively charged and accumulates in cells by intercalating into DNA, which can be monitored by the appearance of bright red fluorescence. $^{33}$

For immunofluorescence detection of Nox-2, p $22^{\text {phox }}$, and $\mathrm{p} 47^{\text {phox }}$, the kidney sections were fixed for $10 \mathrm{~min}$ at $25^{\circ} \mathrm{C}$ in $3.75 \%$ paraformaldehyde, then permeabilized for $10 \mathrm{~min}$ at $25^{\circ} \mathrm{C}$ in $0.2 \%$ Triton $\mathrm{X}-100$. The permeabilized kidney sections were incubated overnight at $4^{\circ} \mathrm{C}$ with a mouse monoclonal antibody against Nox- 2 or a rabbit polyclonal antibody against $\mathrm{p} 47^{\text {phox }}$ or $\mathrm{p} 22^{\text {phox }}$ in PBS supplemented with $3 \%$ goat serum. After washes with PBS, the sections were incubated for $1 \mathrm{~h}$ at $25^{\circ} \mathrm{C}$ in the dark with a goat anti-mouse or a goat anti-rabbit IgG antibody, conjugated with either Alexa Fluor 555 or 488 , and mounted with an anti-fade, water-based mounting medium containing DAPI. The kidney sections were then visualized using the Axioskop2 plus fluorescence microscope.

\section{SOD and CAT Assays}

The animals were anesthetized by intraperitoneal injection of ketamine $(150 \mathrm{mg} / \mathrm{kg})$. Then the animals were perfused through the left ventricle of the heart with $20 \mathrm{ml}$ of PBS. The kidneys were removed and homogenized in ice-cold buffer containing $20 \mathrm{mM}$ HEPES, pH 7.2, $1 \mathrm{mM}$ EGTA, $210 \mathrm{mM}$ mannitol, and $320 \mathrm{mM}$ sucrose. The SOD activity that dismutates superoxide anion to hydrogen peroxide and oxygen was determined using the BIOXYTECH SOD-525 Kit (OXIS International). The CAT activity that converts hydrogen peroxide to water and oxygen was determined using the BIOXYTECH Catalase-520 Kit (OXIS International).

\section{Antioxidant Treatment}

Six-week-old GSD-Ia mice were divided into two groups. The experimental group $(n=4)$ received drinking water supplemented with $2 \mathrm{mM}$ of tempol (Sigma, St Louis, MO, USA) and the control group $(n=4)$ received only water. Both groups of mice were observed for 6 weeks and blood samples were collected every 2 weeks.

\section{Statistical Analysis}

The unpaired $t$-test was performed using the GraphPad Prism Program, version 4 (GraphPad Software, San Diego, CA, USA). Values were considered statistically significant at $P<0.05$.

\section{RESULTS \\ Increased Renal Expression of NADPH Oxidase in GSD-la Mice}

We have previously shown that the Ang/TGF- $\beta 1$ pathway is upregulated in the kidney of GSD-Ia mice. ${ }^{12}$ As this pathway can stimulate the production of ROS by activating NADPH oxidases of the Nox family, ${ }^{14-16}$ we examined the expression of components of NADPH oxidases, including the membrane-associated Nox-2 (gp91 $\left.{ }^{\text {phox }}\right)$, Nox-4, and p22 $2^{\text {phox }}$, and the cytosolic regulatory protein $\mathrm{p} 47^{\text {phox }}$ in the kidneys of GSD-Ia and control mice. To minimize interference from phagocyte NADPH oxidase, blood leukoctyes were removed by perfusion before the study. Western blot analysis showed that renal expression of Nox-2 but not Nox-4 was increased significantly in GSD-Ia mice, compared with the control littermates (Figure 1a). In parallel, the renal expression of the $\mathrm{p} 22^{\text {phox }}$ and $\mathrm{p} 47^{\text {phox }}$ subunits was also upregulated in GSD-Ia kidneys (Figure 1a).

Immunofluoresence analysis confirmed the increase in the expression of membrane-associated Nox- 2 and $\mathrm{p} 22^{\text {phox }}$ in the GSD-Ia kidneys relative to wild-type kidneys (Figure 1b), consistent with the western blot results. Immunofluoresence analysis also showed that the expression and membrane association of $\mathrm{p} 47^{\mathrm{phox}}$ was markedly increased in the GSD-Ia kidneys (Figure 1b), consistent with the western blot results and the activation of the NADPH oxidase activity triggered by $47^{\text {phox }}$ membrane translocation. ${ }^{34,35}$

\section{Activation of the Akt/FoxO Pathway in GSD-la Kidneys} Both Ang $\mathrm{II}^{22,23}$ and TGF- $\beta 1^{24,25}$ activate the serine/threonine kinase, Akt/protein kinase B. ${ }^{26}$ In the kidneys of GSD-Ia mice, western blot analysis showed that Ser473 in Akt was phosphorylated at much higher levels than the control littermates (Figure 2a). Interestingly, the total amount of Akt was higher in the GSD-Ia kidneys compared with controls (Figure 2a), suggesting that in addition to an increased level of activation there may also be an increased expression level of Akt.

Akt regulates the production of ROS through phosphoryl inactivation of the FoxO family of transcription factors. ${ }^{27,28}$ Western blot analysis showed that although the overall levels of renal $\mathrm{FoxO} 1$ and $\mathrm{FoxO} 3$ proteins remain unchanged 
a

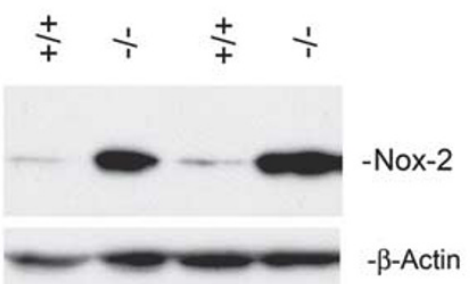

$\frac{1}{+}+\frac{1}{+}$

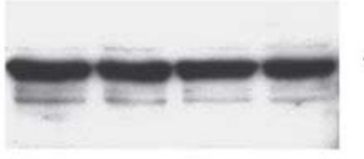

- Nox4

$-\beta$-Actin
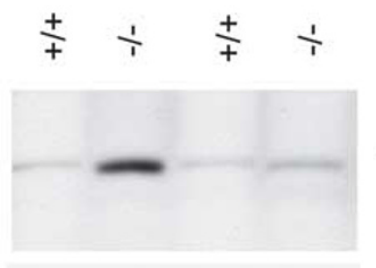

$-\beta$-Actin

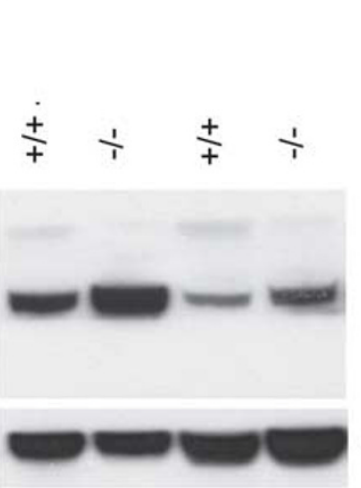

$-p 47^{\text {phox }}$

$-\beta$-Actin b
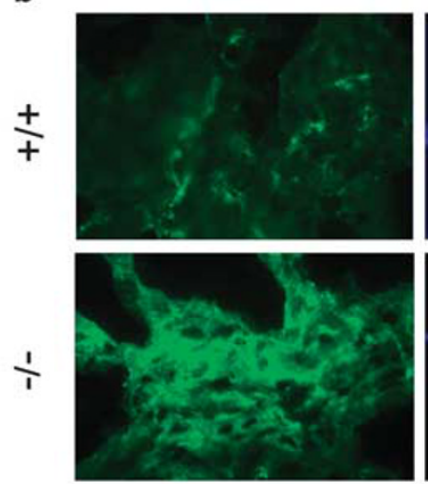

p22 $2^{\text {phox }}$
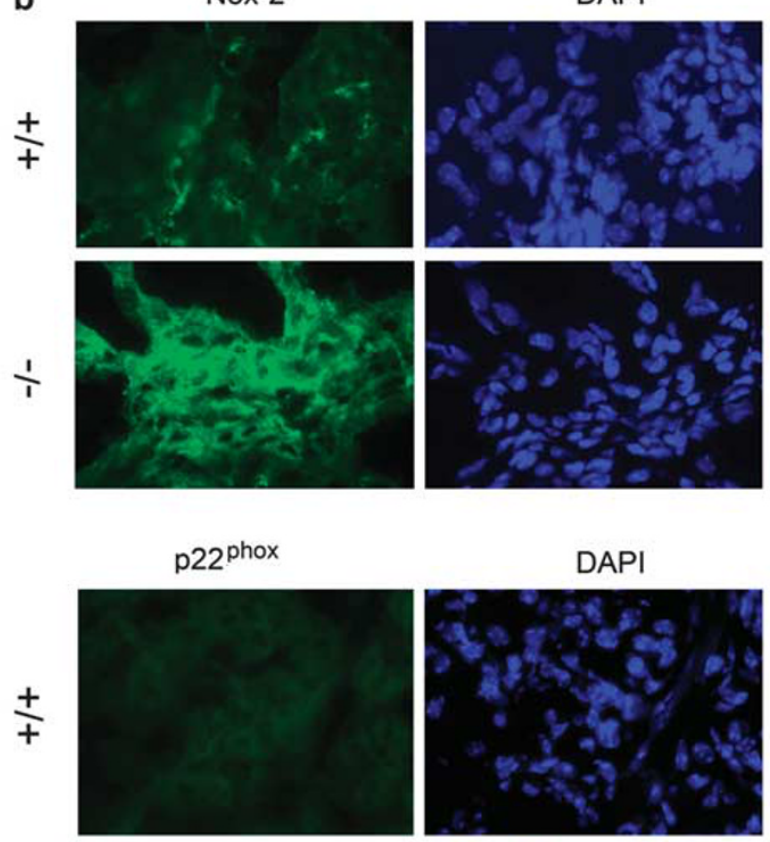

DAPI
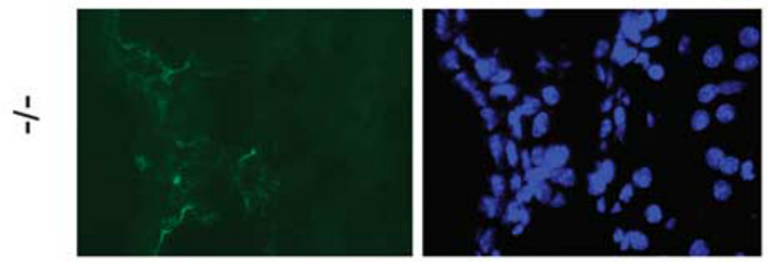

DAPI
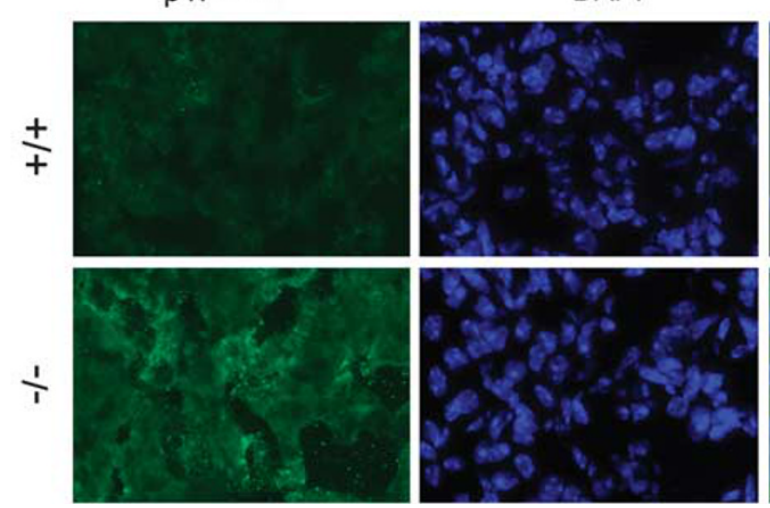
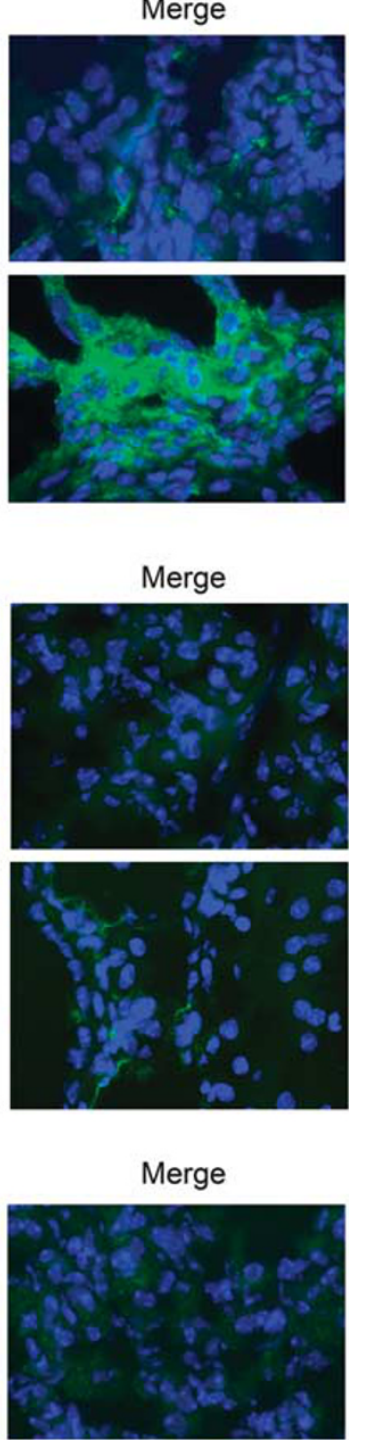

Figure 1 Analysis of renal expression of the NADPH oxidase in GSD-la mice. (a) Western blot analysis of protein extracts of perfused kidneys in 6-week-old wild-type $(+/+, n=4)$ and GSD-la $(-/-, n=4)$ mice using antibodies against Nox-2, Nox-4, p22 ${ }^{\text {phox }}$ p47 ${ }^{\text {phox }}$, or $\beta$-actin. Data from two pairs of littermates are shown and each lane contains $50 \mu \mathrm{g}$ protein. (b) Representative immunofluorescence analysis of Nox-2 (green fluorescence), p2 $2^{\text {phox }}$ (green fluorescence), or $\mathrm{p} 47^{\text {phox }}$ (green fluorescence) and DAPI nuclei staining (blue fluorescence) in kidney sections from 6-week-old wild-type $(+/+)$ and GSD-la (-/-) mice at magnifications of $\times 1000$.

between GSD-Ia and controls (Figure 2b), the levels of phosphorylated FoxO proteins are significantly different with p-FoxO1 (Ser256), p-FoxO1 (Thr24), p-FoxO3a (Thr32), p-FoxO3a (Ser253), and p-FoxO4 (Ser193), all upregulated in GSD-Ia mice compared with littermate controls (Figure 2b).

When the phosphorylated FoxO proteins are bound by the adaptor proteins, it triggers the exclusion of the
FoxO proteins from the nucleus and the loss of transcriptional activity. ${ }^{27,28}$ Immunohistochemical analysis using an antibody to FoxO3a phosphorylated at Ser253 showed markedly increased staining in the kidney of GSD-Ia mice compared with the control littermates (Figure 2c), with the majority of the stain cytoplasmic, consistent with nuclear exclusion. 

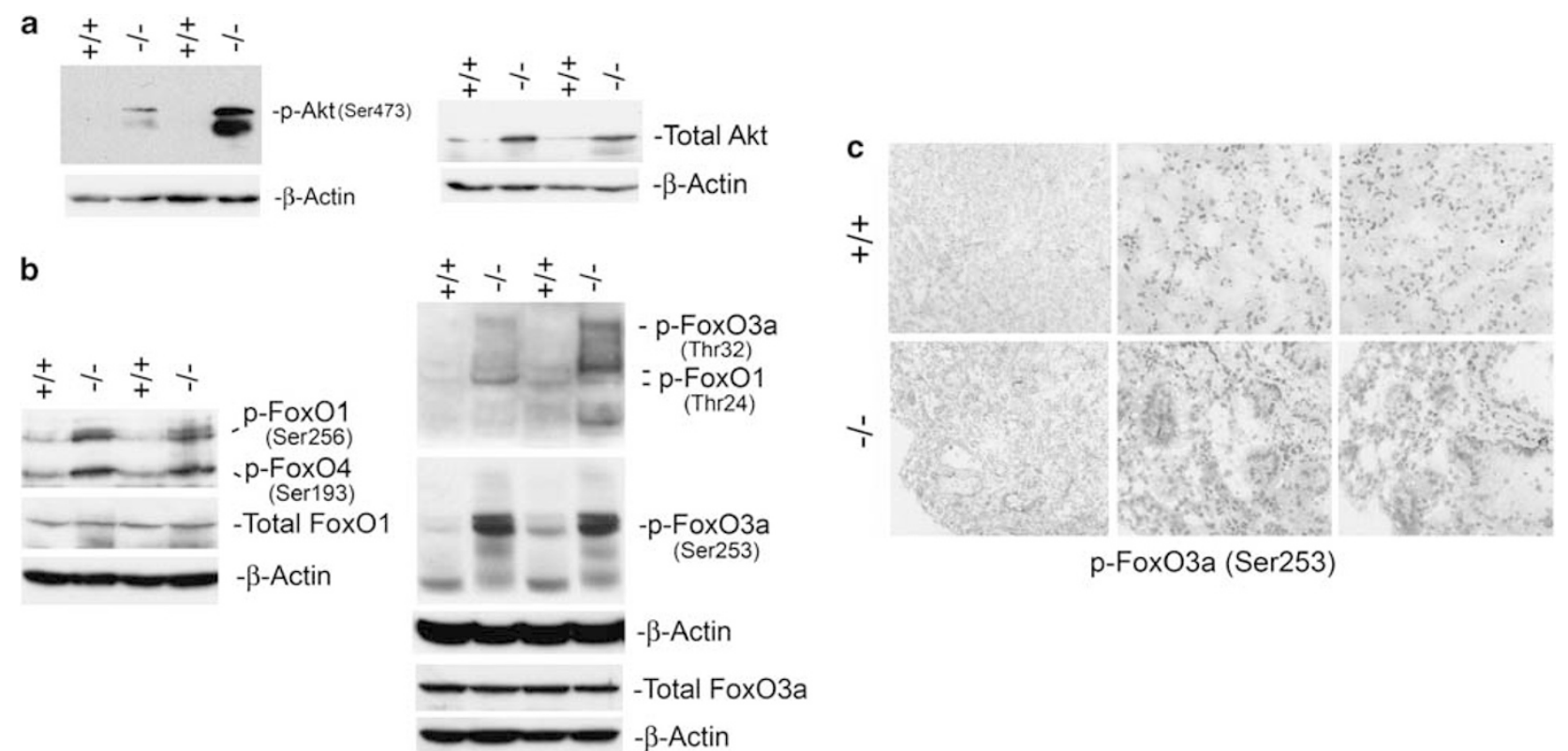

Figure 2 Analysis of renal expression of Akt and FoxO proteins in GSD-la mice. (a) Western blot analysis of renal extracts from 6-week-old wild-type $(+/+, n=4)$ and GSD-la $(-/-, n=4)$ mice using antibodies against phospho-Akt (Ser473), total Akt, or $\beta$-actin. Data from two pairs of littermates are shown and each lane contains $100 \mu \mathrm{g}$ protein. (b) Western blot analysis of renal extracts in 6-week-old wild-type $(+/+, n=4)$ and GSD-la $(-/-$, $n=4)$ mice using antibodies against phospho-FoxO1 (Ser256)/FoxO4 (Ser193), total FoxO1, phospho-FoxO1 (Thr24)/FoxO3a (Thr32), phospho-FoxO3a (Ser253), total FoxO3a, or $\beta$-actin. Data from two pairs of littermates are shown and each lane contains $200 \mu \mathrm{g}$ protein. (c) Representative immunohistochemical analysis of p-FoxO3a (Ser253). Plates shown are kidney sections from 6-week-old wild-type (+/ +) and GSD-la (-/-) mice at magnifications of $\times 100, \times 400$, and $\times 400$.

Reduced Expression of SOD and CAT in GSD-la Kidneys Inactivation of FoxO transcription factors can repress expression of SOD and CAT, which scavenge oxygen-free radicals. ${ }^{29-31}$ Quantitative real-time RT-PCR analysis showed that renal SOD1, SOD2, and SOD3 mRNA levels in 4- to 6-week-old GSD-Ia mice are on average 60, 73, and 59\%, respectively, of the levels in their age-matched control littermates (Figure 3a). Western blot analysis confirmed the decrease in renal SOD1 and SOD2 proteins (Figure 3b). In contrast, SOD3 protein expression was unaffected despite a loss of SOD3 mRNA expression in GSD-Ia mice. Activity assays showed that renal SOD activities in 4- and 6-week-old GSD-Ia mice were 41.5 and $54.1 \%$, respectively, of the activities in their age-matched control mice (Figure 3c).

Quantitative real-time RT-PCR analysis showed that renal CAT mRNA levels in 4- to 6-week-old GSD-Ia mice are on average $54 \%$ of the levels in their age-matched control littermates (Figure 3d). Western blot analysis confirmed the decrease in CAT proteins in the kidneys of GSD-Ia mice (Figure 3e). Consistent with this, activity assays showed that renal CAT activities in 4- and 6-week-old GSD-Ia mice were 35.3 and $62.2 \%$, respectively, of the activities in their agematched control mice ((Figure 3f).

\section{Increased Oxidative Stress in GSD-la Kidneys}

With an increase in the expression of NADPH oxidase and a decrease in the expression of both SOD and CAT in the kidney of GSD-Ia mice, there could be an excess production of renal ROS, which would alter the renal redox balance, resulting in oxidative damage. We therefore examined ROS production in the kidney using the reporter molecule DHE. Oxidation of DHE results in the production of ethidium, which will bind to DNA to produce a bright red fluorescence. $^{33}$ Kidney sections from GSD-Ia and control mice were incubated with DHE, and its conversion to fluorescent DNA-bound ethidium was examined by immunofluorescence analysis. The GSD-Ia kidney sections showed significantly stronger nuclear fluorescent signals, evident by the colocalization of the fluorescent DNA-bound ethidium with DAPI nuclear staining (Figure 4a), as compared with the control kidney sections, consistent with renal oxidative stress in the GSD-Ia mice.

One of the biochemical consequences of elevated ROS is DNA-strand breakage, ${ }^{36,37}$ which results in the production of $8-\mathrm{OHdG} .{ }^{38}$ Immunohistochemical analysis showed that there was a very low level of 8-OHdG immunostaining in wild-type kidneys (Figure 4b). However, in the GSD-Ia kidneys, we observed intense 8-OHdG staining in the glomeruli and interstitial tissues (Figure $4 \mathrm{~b}$ ).

\section{The Antioxidant Tempol Improves Renal Function in GSD-la Mice}

To evaluate renal function in GSD-Ia mice, we measured the levels of serum BUN in 6-week-old control and GSD-Ia mice. 

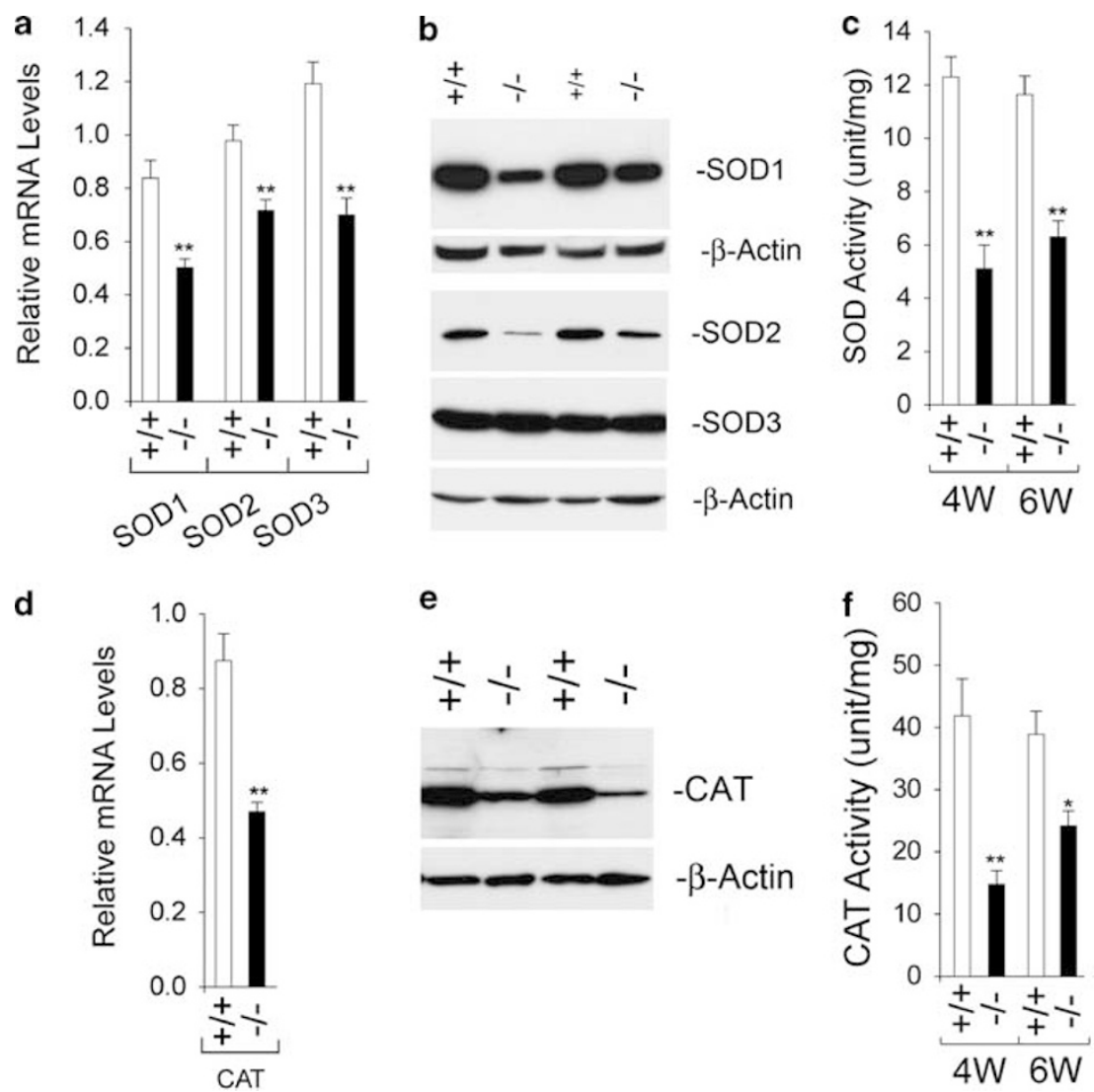

Figure 3 Suppression of renal expression of SOD and CAT in GSD-la mice. The renal expression of antioxidant enzymes was examined in 4-week-old $(4 \mathrm{~W})$ and 6-week-old (6W) wild-type $(+/+)$ and GSD-la $(-/-)$ mice. (a) Quantification of SOD1, SOD2, SOD3 mRNA in the kidneys of 4- to 6-week-old control and GSD-la mice by real-time RT-PCR. Results are the mean \pm s.e.m. Each point represents the average of 12 animals. (b) Western blot analysis of renal extracts from 6-week-old control $(n=6)$ and GSD-la $(n=6)$ mice using antibodies against SOD1, SOD2, SOD3, or $\beta$-actin. Data from two pairs of littermates are shown and each lane contains $50 \mu \mathrm{g}$ protein. (c) Quantification of SOD enzymatic activity in the kidneys of 4- or 6-week-old control and GSD-la mice. Each point represents the average of eight animals. (d) Quantification of CAT mRNA in the kidneys of 4- to 6-week-old control and GSD-la mice by real-time RT-PCR. Results are the mean \pm s.e.m. Each point represents the average of 12 animals. (e) Western blot analysis of renal extracts from 6-week-old control $(n=6)$ and GSD-la $(n=6)$ mice using antibodies against CAT or $\beta$-actin. Data from two pairs of littermates are shown and each lane contains $50 \mu \mathrm{g}$ protein. (f) Quantification of CAT enzymatic activity in the kidneys of 4- or 6-week-old control and GSD-la mice. Each point represents the average of eight animals. ${ }^{*} P<0.05 ;{ }^{*} P<0.005$.

Serum BUN levels in GSD-Ia mice were 40\% higher than those in the control littermates (Figure 5a), indicating that renal function in GSD-Ia mice was compromised.

Tempol is a small, cell membrane permeable SOD mimetic that attenuates superoxide anion production. ${ }^{39}$ Therefore, if ROS elevation and damage is contributing to renal damage in GSD-Ia, tempol treatment may improve renal function. To study this, we treated 6-week-old GSD-Ia mice for 6 weeks with tempol and monitored renal function by measuring the levels of serum BUN before and after 4- and 6 weeks of treatment. To account for individual variations, all data are expressed relative to the measurements made at age 6 weeks before initiation of tempol therapy. The vehicle-treated GSD-Ia mice were used as controls.

The serum levels of BUN in wild-type mice were more or less unchanged between age 6 and 12 weeks (data not shown). Following 4-6 weeks of vehicle treatment, the relative BUN levels in GSD-Ia mice increased to $146 \%$ of the levels at age 6 weeks (Figure 5b), suggesting continued deterioration in renal function. On the other hand, after 4- to 6 weeks of tempol treatment, the relative BUN levels in GSD-Ia mice were $85 \%$ relative to the levels at age 6 weeks (Figure 5b). In support of this, western blot analysis showed that although renal CAT protein expression was still low following 6 weeks of vehicle treatment of GSD-Ia mice, in tempol-treated GSD-Ia mice, CAT expression was comparable to that in the age-matched wild-type mice (Figure 5c).

We have previously shown that the kidneys of 6-week-old GSD-Ia mice exhibit excessive glycogen storage, tubular atrophy, tubular dilation, increased Bowman's capsule spaces, and multifocal interstitial fibrosis. ${ }^{12}$ Histological examination of the kidneys in 12-week-old vehicle- and tempol-treated GSD-Ia mice again showed similar histological abnormalities 

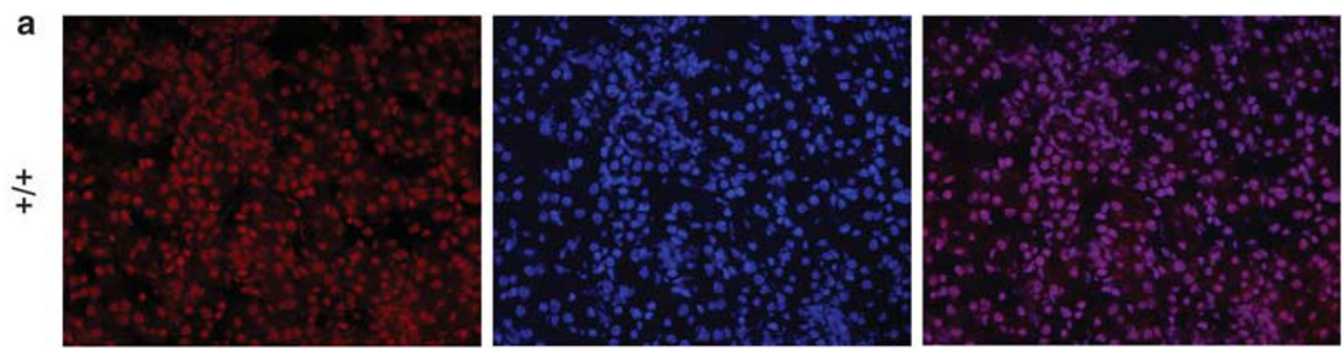

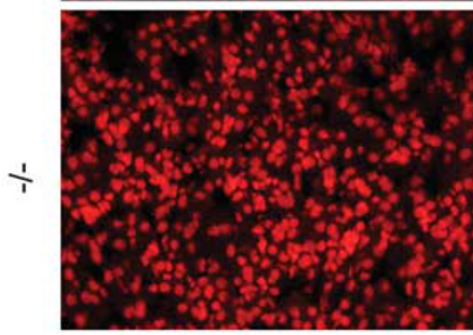

DHE

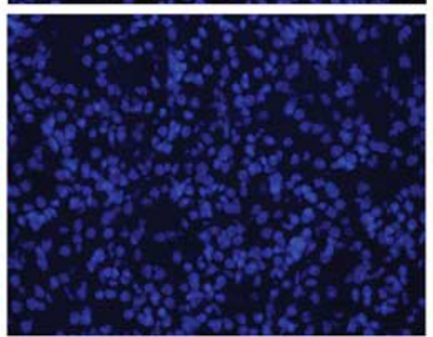

DAPI

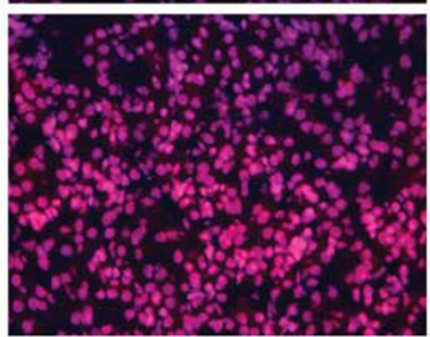

Merge
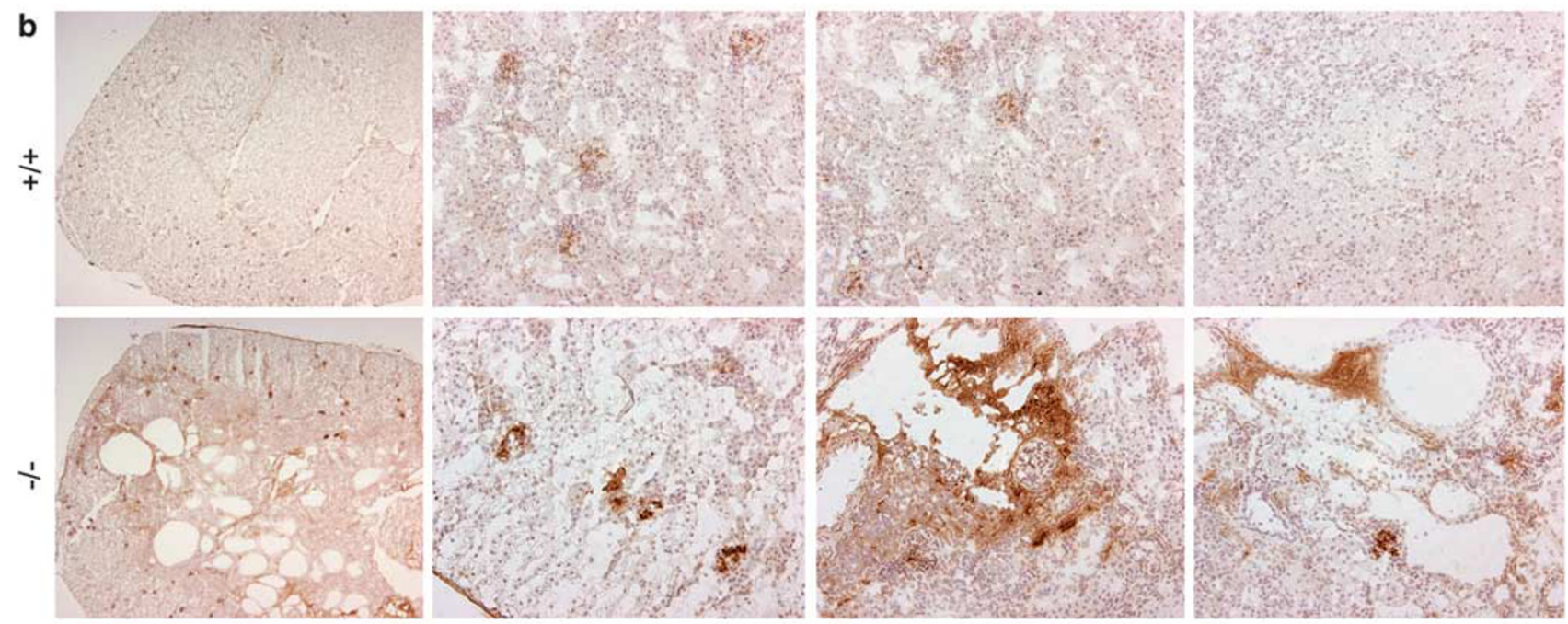

8-OH-dG

Figure 4 Enhanced oxidative stress in the kidneys of GSD-la mice. (a) Representative immunofluorescence of oxidized DHE staining (red fluorescence) and DAPI nuclei staining (blue fluorescence) in kidney sections from 6-week-old wild-type $(+/+, n=4)$ and GSD-la $(-/-, n=4)$ mice at magnifications of $\times 400$. The nuclear colocalization of DHE with DAPI staining was indicated by purple in the merged image. (b) Representative immunohistochemical analysis of 8-OHdG staining in the kidney sections from 6-week-old wild-type $(+/+, n=4)$ and GSD-la $(-/-, n=4)$ mice at magnifications from left to right, $\times 25, \times 200, \times 200$, and $\times 200$.

(Figure 6). However, the vehicle-treated GSD-Ia mice showed increased renal damage, characterized by marked tubular dilation and increased Bowman's capsule spaces (Figure 6a). Moreover, Masson's trichrome staining revealed more pronounced renal fibrosis in vehicle-treated GSD-Ia mice as compared with tempol-treated GSD-Ia mice (Figure 6b). For quantitative histochemical measurement of renal fibrosis, collagen was imaged using von Gieson stain and converted into pixel density units using Adobe Photoshop. Results in Figure $6 \mathrm{~b}$ showed that the density units in the kidneys of the vehicle-treated GSD-Ia mice were 3.2-fold higher than those in the tempol-treated GSD-Ia mice, confirming the improvement in renal pathology after tempol treatment.
Taken together, these results indicate that tempol treatment of GSD-Ia mice improved renal function and delayed renal damage and fibrosis.

\section{DISCUSSION}

GSD-Ia patients under intensive dietary therapy continue to suffer from the long-term complications of renal disease ${ }^{4-6}$ but the underlying mechanisms remain to be elucidated. We have previously shown that the Ang II/TGF- $\beta 1$ pathway is upregulated in the GSD-Ia kidney and mediates renal fibrosis in murine GSD-Ia. ${ }^{12}$ However, the molecular mechanisms leading to renal dysfunctions in GSD-Ia are almost certain to involve multiple pathways, suggesting that additional conse- 
quences of the Ang II/TGF- $\beta 1$ pathway ${ }^{14-16,22-25}$ may also be important. The pathway is also a mediator of oxidative stress, having roles in both promoting ROS production and reducing ROS detoxification. In this study, we investigated whether the Ang II/TGF- $\beta 1$ pathway is causing oxidative stress in the kidney of GSD-Ia mice and, if so, the mechanism behind these processes.

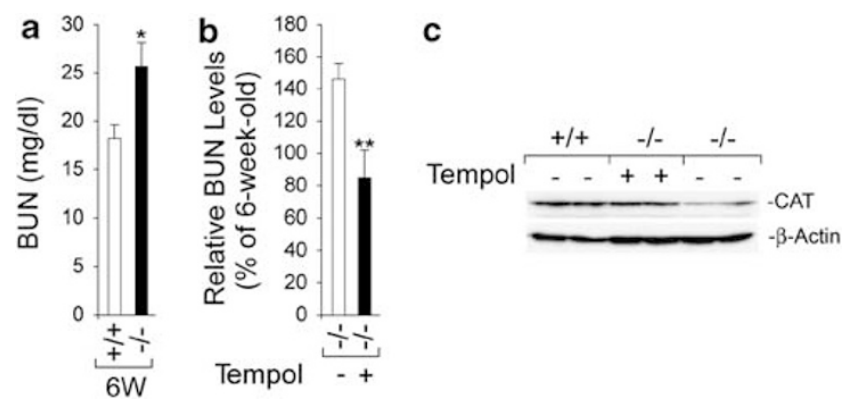

Figure 5 Improved renal function in GSD-la mice after tempol treatment. (a) Serum levels of BUN in 6-week-old (6W) wild-type $(+/+; n=9)$ and GSD-la $(-/-; n=9)$ mice. ${ }^{*} P<0.05$. (b) Relative serum levels of BUN in 10- and 12-week-old GSD-la (-/-) mice after 4- to 6-week tempol (+; $n=8)$ or vehicle $(-; n=8)$ treatment. ${ }^{* * P}<0.005$. (c) Western blot analysis of protein extracts of perfused kidneys in 12-week-old wild-type $(+/+$, $n=4)$ and GSD-la $(-/-)$ mice after 6 weeks of treatment with tempol $(+, n=4)$ or vehicle $(-, n=4)$, using antibodies against CAT or $\beta$-actin. Data from two mice are shown for each treatment, and each lane contains $50 \mu \mathrm{g}$ protein.
Although phagocytes use ROS as part of the innate immunity against pathogens, ${ }^{40}$ both Ang $\mathrm{II}^{14,15}$ and TGF- $\beta 1,{ }^{16}$ can stimulate intracellular formation of ROS in non-phagocytic cells by activating NADPH oxidases. We show that renal expression of Nox-2, p $22^{\text {phox }}$ and $\mathrm{p} 47^{\text {phox }}$, proteins are increased in the kidney of GSD-Ia mice compared with the controls, consistent with a higher level of ROS production in the GSD-Ia kidney. To control intracellular ROS levels, the Nox family is counter-regulated by the antioxidant enzymes SOD $^{29,30}$ and CAT. $^{31}$ This control pathway is mediated by the FoxO transcription factors, ${ }^{27,28}$ which among other activities, upregulate expression of the SOD and CAT genes, leading to ROS metabolism. In turn, the FoxO proteins are regulated by the serine/threonine kinase Akt. $^{26}$ When phosphorylated at Ser $473,{ }^{41}$ Akt can phosphorylate FoxO members. ${ }^{42}$ This results in FoxO proteins binding to the chaperone molecule 14-3-3 and the translocation of the resulting FoxO complex out of the nucleus, ${ }^{27,28}$ where it can no longer exert a regulatory effect on the expression of the SOD and CAT genes, essentially suppressing the production of these antioxidant enzymes. We show that the Akt/FoxO signaling pathway is also activated, with the levels of p-Akt (Ser473) and total Akt increased in GSD-Ia kidneys relative to controls. Although the overall levels of FoxO proteins were not elevated, the level of phosphorylated FoxO1 on Thr24 and Ser 256, and FoxO3a and FoxO4 on equivalent sites were all upregulated in the kidney of GSD-Ia mice compared with the controls.
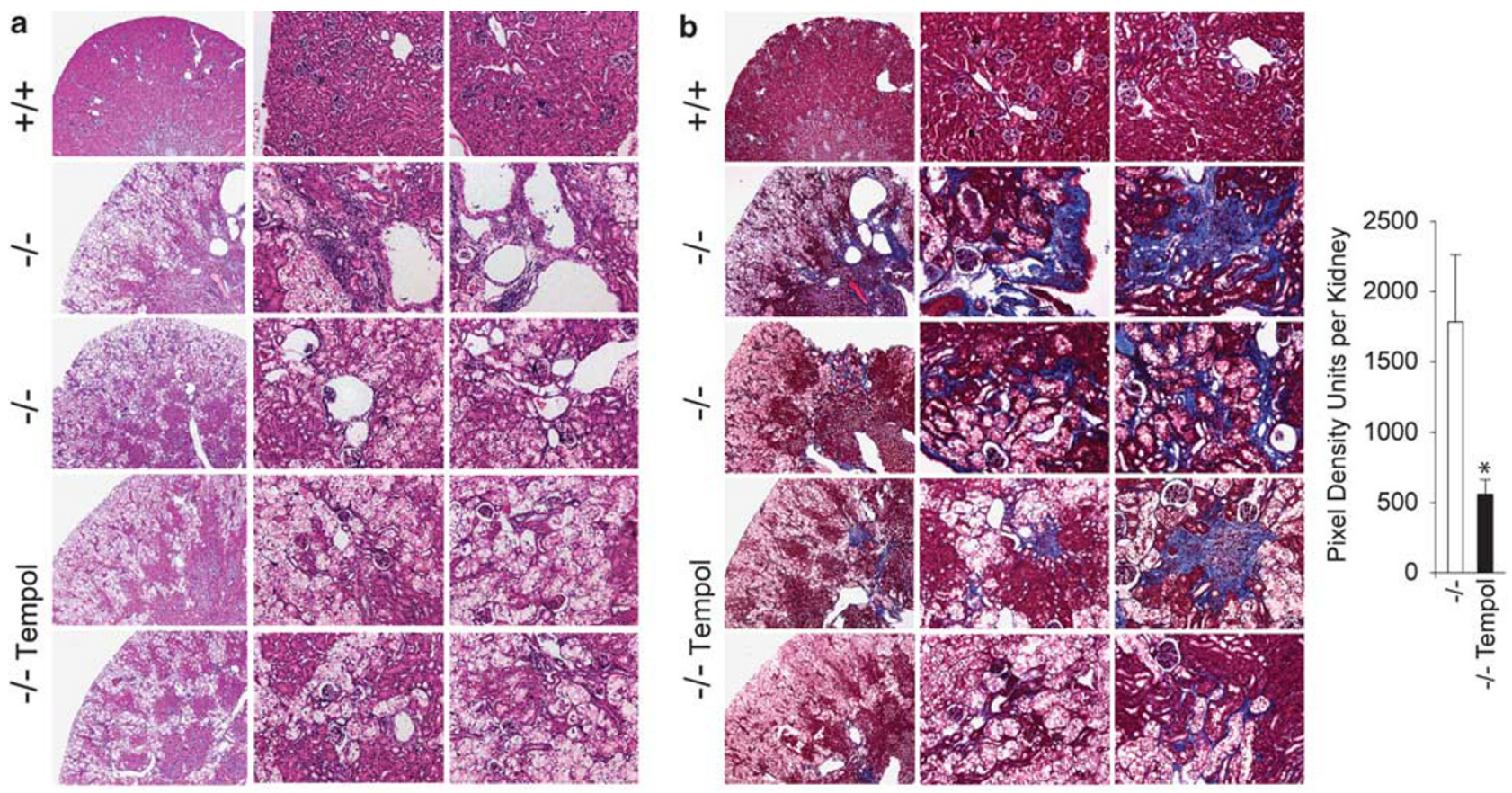

Figure 6 Histological analyses of the kidneys in tempol- or vehicle-treated GSD-la mice. (a) H\&E analyses. (b) Masson's trichrome staining and quantification of renal fibrosis by von Gieson staining. Plates show kidney sections from 12-week-old wild-type $(+/+, n=4)$ and GSD-la $(-/-)$ mice after 6 -week of vehicle $(-/-, n=4)$ or tempol $(-/-$ Tempol, $n=4)$ treatment at magnifications from left to right, $\times 50, \times 200$, and $\times 200$. Data from two vehicle-treated and two tempol-treated GSD-la mice are shown. Interstitial fibrosis is shown by the blue colored Masson's trichrome staining of the collagen fibers and by quantification of von Gieson staining. Each point represents the average of four animals. Values represent mean \pm s.e.m. ${ }^{\star} P<0.05$. 
Consistent with this, we show that renal expression of SOD and CAT are downregulated at both the protein and activity levels in GSD-Ia mice, relative to their control littermates. As expected, this combination of increased NADPH oxidase activity and decreased SOD and CAT activity resulted in an increase in ROS. Oxidative stress was markedly increased in the kidneys of GSD-Ia mice, relative to their controls, as shown by the increase in both the oxidation of DHE and oxidative damage of DNA. Moreover, treatment with SOD mimetic tempol reversed the functional differences seen between the wild-type and GSD-Ia kidneys, consistent with the reversal of the intracellular ROS load and consequent reduction of oxidative damage.

Renal disease in GSD-Ia shows a similar clinical course and pathology to diabetic nephropathy. ${ }^{43}$ Both dysfunctions start with a long period of silent disease during which glomerular hyperfiltration is the only demonstrable renal abnormality. This is followed by the development of microalbuminuria, proteinuria, and ultimately, renal failure. Pathologically, both show interstitial fibrosis, tubular atrophy, and glomerulosclerosis with marked glomerular basement-membrane thickening. In an earlier study, we showed that Ang II/TGF$\beta 1$ mediates renal fibrosis in GSD- $\mathrm{Ia}^{12}$ in a manner similar to that seen in diabetes. ${ }^{44,45}$ We now extend this observation by showing that similar mechanisms of oxidative stress that mediate diabetic nephropathy ${ }^{46,47}$ also mediate nephropathy in GSD-Ia. Prior to our studies, an Ang-converting enzyme (ACE) inhibitor therapy for renal disease had been evaluated in a 10-year retrospective study with a cohort of 95 GSD-Ia patients. ${ }^{48}$ That study showed that the progression from glomerular hyperfiltration to microalbuminuria was significantly delayed by ACE inhibitors. However, the progression from microalbuminuria to proteinuria was not improved by the ACE inhibitors. These findings suggested that although ACE-inhibitor therapy could be beneficial, there was a need for additional therapeutic approaches for the longer term treatment of GSD-Ia. Our demonstration that antioxidant treatment normalizes serum levels of BUN, improves renal CAT expression, and delays renal damage and fibrosis suggests that antioxidant therapy may also be beneficial for the renal dysfunction in GSD-Ia.

In conclusion, we have investigated a murine model of GSD-Ia, which accurately reflects the metabolic aspects of the human disease, to elucidate potential mechanisms underlying the renal dysfunction. We have shown that GSD-Ia kidney exhibit enhanced oxidative stress that is mediated by activation of NADPH oxidase and suppression of antioxidant enzymes, SOD and CAT, mediated by the Akt/FoxO pathway. As the mice have proven to be a faithful model of human GSD-Ia, our findings suggest that similar mechanisms might be expected in the human GSD-Ia disorder.

\section{ACKNOWLEDGEMENT}

This research was supported by the Intramural Research Program of the $\mathrm{NICHD}, \mathrm{NIH}$.

\section{DISCLOSURE/CONFLICT OF INTEREST}

The authors declare no conflict of interest.

1. Chou JY, Matern D, Mansfield BC, et al. Type I glycogen storage diseases: disorders of the glucose-6-phosphatase complex. Curr Mol Med 2002;2:121-143.

2. Greene $H L$, Slonim $A E, O^{\prime}$ Neill Jr JA, et al. Continuous nocturnal intragastric feeding for management of type 1 glycogen-storage disease. N Engl J Med 1976;294:423-425.

3. Chen YT, Cornblath M, Sidbury JB. Cornstarch therapy in type I glycogen storage disease. N Engl J Med 1984;310:171-175.

4. Chen YT, Coleman RA, Scheinman Jl, et al. Renal disease in type I glycogen storage disease. N Engl J Med 1988;318:7-11.

5. Verani R, Bernstein J. Renal glomerular and tubular abnormalities in glycogen storage disease type I. Arch Pathol Lab Med 1988;112: 271-274.

6. Baker L, Dahlem S, Goldfarb S, et al. Hyperfiltration and renal disease in glycogen storage disease, type I. Kidney Int 1989;35:1345-1350.

7. Weinstein DA, Somers MJ, Wolfsdorf Jl. Decreased urinary citrate excretion in type 1a glycogen storage disease. J Pediatr 2001;138: 378-382.

8. Rake JP, Visser G, Labrune P, et al. Glycogen storage disease type I: diagnosis, management, clinical course and outcome. Results of the European Study on Glycogen Storage Disease Type I (ESGSD I). Eur J Pediatr 2002;161(Suppl 1):S20-S34.

9. Lei KJ, Chen H, Pan CJ, et al. Glucose-6-phosphatase dependent substrate transport in the glycogen storage disease type 1a mouse. Nat Genet 1996;13:203-209.

10. Ruiz-Ortega M, Ruperez M, Esteban V, et al. Angiotensin II: a key factor in the inflammatory and fibrotic response in kidney diseases. Nephrol Dial Transplant 2006;21:16-20.

11. Wolf $G$. Renal injury due to renin-angiotensin-aldosterone system activation of the transforming growth factor-beta pathway. Kidney Int 2006;70:1914-1919.

12. Yiu WH, Pan C-J, Ruef RA, et al. Angiotensin mediates renal fibrosis in the nephropathy of glycogen storage disease type la. Kidney Int 2008;73:716-723.

13. Bottinger EP, Bitzer M. TGF-beta signaling in renal disease. J Am Soc Nephrol 2002;13:2600-2610.

14. Sachse A, Wolf $G$. Angiotensin Il-induced reactive oxygen species and the kidney. J Am Soc Nephrol 2007;18:2439-2446.

15. Welch WJ. Angiotensin II-dependent superoxide: effects on hypertension and vascular dysfunction. Hypertension 2008;52:51-56.

16. Lee $H B, Y u M R$, Yang $Y$, et al. Reactive oxygen species-regulated signaling pathways in diabetic nephropathy. J Am Soc Nephrol 2003;14(Suppl 3):S241-S245.

17. Abid MR, Razzaque MS, Taguchi T. Oxidant stress in renal pathophysiology. Contrib Nephrol 2005;148:135-153.

18. Singh D, Kaur R, Chander V, et al. Antioxidants in the prevention of renal disease. J Med Food 2006;9:443-450.

19. Shah SV, Baliga R, Rajapurkar M, et al. Oxidants in chronic kidney disease. J Am Soc Nephrol 2007;18:16-28.

20. Lambeth JD. NOX enzymes and the biology of reactive oxygen. Nat Rev Immunol 2004;4:181-189.

21. Gill PS, Wilcox CS. NADPH oxidases in the kidney. Antioxid Redox Signal 2006;8:1597-1607.

22. Gorin Y, Kim NH, Feliers D, et al. Angiotensin II activates Akt/protein kinase $B$ by an arachidonic acid/redox-dependent pathway and independent of phosphoinositide 3-kinase. FASEB J 2001;15: 1909-1920.

23. Block K, Ricono JM, Lee DY, et al. Arachidonic acid-dependent activation of a p22(phox)-based NAD(P)H oxidase mediates angiotensin II-induced mesangial cell protein synthesis and fibronectin expression via Akt/PKB. Antioxid Redox Signal 2006;8:1497-1508.

24. Runyan CE, Schnaper HW, Poncelet AC. The phosphatidylinositol 3-kinase/Akt pathway enhances Smad3-stimulated mesangial cell collagen I expression in response to transforming growth factor-beta1. J Biol Chem 2004;279:2632-2639.

25. Kato M, Yuan H, Xu ZG, et al. Role of the Akt/FoxO3a pathway in TGF-beta1-mediated mesangial cell dysfunction: a novel mechanism related to diabetic kidney disease. J Am Soc Nephrol 2006;17: 3325-3335. 
26. Manning BD, Cantley LC. AKT/PKB signaling: navigating downstream. Cell 2007;129:1261-1274.

27. Lam EW, Francis RE, Petkovic M. FOXO transcription factors: key regulators of cell fate. Biochem Soc Trans 2006;34:722-726.

28. Huang $\mathrm{H}$, Tindall DJ. Dynamic FoxO transcription factors. J Cell Sci 2007;120:2479-2487.

29. Johnson F, Giulivi C. Superoxide dismutases and their impact upon human health. Mol Aspects Med 2005;26:340-352.

30. Afonso V, Champy R, Mitrovic D, et al. Reactive oxygen species and superoxide dismutases: role in joint diseases. Joint Bone Spine 2007;74:324-329.

31. Kirkman HN, Gaetani GF. Mammalian catalase: a venerable enzyme with new mysteries. Trends Biochem Sci 2007;32:44-50.

32. Venkatesan B, Mahimainathan L, Das F, et al. Downregulation of catalase by reactive oxygen species via PI 3 kinase/Akt signaling in mesangial cells. J Cell Physiol 2007;211:457-467.

33. Laurindo FR, Fernandes DC, Santos CX. Assessment of superoxide production and NADPH oxidase activity by HPLC analysis of dihydroethidium oxidation products. Methods Enzymol 2008;441:237-260.

34. Kitada M, Koya D, Sugimoto $T$, et al. Translocation of glomerular p47phox and p67phox by protein kinase C-beta activation is required for oxidative stress in diabetic nephropathy. Diabetes 2003;52: 2603-2614.

35. Tojo A, Asaba K, Onozato ML. Suppressing renal NADPH oxidase to treat diabetic nephropathy. Expert Opin Ther Targets 2007;11:1011-1018.

36. Orrenius S, Gogvadze V, Zhivotovsky B. Mitochondrial oxidative stress: implications for cell death. Annu Rev Pharmacol Toxicol 2007;47: 143-183.

37. Dröge W. Free radicals in the physiological control of cell function. Physiol Rev 2002;82:47-95.
38. Pilger A, Rüdiger HW. 8-Hydroxy-2'-deoxyguanosine as a marker of oxidative DNA damage related to occupational and environmental exposures. Int Arch Occup Environ Health 2006;80:1-15.

39. Augusto O, Trindade DF, Linares E, et al. Cyclic nitroxides inhibit the toxicity of nitric oxide-derived oxidants: mechanisms and implications. An Acad Bras Cienc 2008;80:179-189.

40. Rada B, Leto TL. Oxidative innate immune defenses by Nox/Duox family NADPH oxidases. Contrib Microbiol 2008;15:164-187.

41. Jacinto $E$, Facchinetti V, Liu D, et al. SIN1/MIP1 maintains rictor-mTOR complex integrity and regulates Akt phosphorylation and substrate specificity. Cell 2006;127:125-137.

42. Tran H, Brunet A, Griffith EC, et al. The many forks in FOXO's road. Sci STKE 2003;2003:RE5.

43. Mundy HR, Lee PJ. Glycogenosis type I and diabetes mellitus: a common mechanism for renal dysfunction? Med Hypotheses 2002;59:110-114.

44. Schrijvers BF, De Vriese AS, Flyvbjerg A. From hyperglycemia to diabetic kidney disease: the role of metabolic, hemodynamic, intracellular factors and growth factors/cytokines. Endocr Rev 2004;25:971-1010.

45. Wolf $\mathrm{G}$. New insights into the pathophysiology of diabetic nephropathy: from haemodynamics to molecular pathology. Eur J Clin Invest 2004;34:785-796.

46. Maritim AC, Sanders RA, Watkins 3rd JB. Diabetes, oxidative stress, and antioxidants: a review. J Biochem Mol Toxicol 2003;17:24-38.

47. Forbes JM, Coughlan MT, Cooper ME. Oxidative stress as a major culprit in kidney disease in diabetes. Diabetes 2008;57:1446-1454.

48. Melis D, Parenti G, Gatti R, et al. Efficacy of ACE-inhibitor therapy on renal disease in glycogen storage disease type 1: a multicentre retrospective study. Clin Endocrinol (Oxf) 2005;63:19-25. 